\title{
Dehalobium species implicated in 2,3,7,8-tetrachloro-p-dioxin dechlorination in the contaminated sediments of Sydney Harbour Estuary
} Matthew Lee ${ }^{1}$, Gan Liang1, Sophie I. Holland ${ }^{1}$, Casey O’Farrell², Keith Osbourne ${ }^{3}$, Michael J. Manefield ${ }^{1}$

${ }^{1}$ UNSW Water Research Centre, School of Civil and Environmental Engineering, UNSW, Sydney, NSW 2052, Australia

${ }^{2}$ Coffey Tetra Tech, Southbank Victoria 3006, Australia

${ }^{3}$ Environment Protection Science Branch, Department of Planning, Industry \& Environment, Lidcombe, NSW, 2141

\section{Abstract}

Polychlorinated dibenzo- $p$-dioxins and furans (PCDD/F) are some of the most environmentally recalcitrant and toxic compounds. They are naturally occurring and by-products of anthropogenic activity. Sydney Harbour Estuary (Sydney, Australia), is heavily contaminated with PCDD/F. Analysis of sediment cores revealed that the contamination source in Homebush Bay continues to have one of the highest levels of PCDD/F contamination in the world (5207 pg WHO-TEQ $\mathrm{g}^{-1}$ ) with $>50 \%$ of the toxicity attributed to 2,3,7,8-tetrachlorodibenzo- $p$-dioxin $(2,3,7,8-T C D D)$ the most toxic and concerning of the PCDD/F congeners. Comparison of congener profiles at the contamination source with surrounding bays and historical data provided evidence for the attenuation of 2,3,7,8-TCDD and other congeners at the source. This finding was supported by the detection of di-, mono- and unchlorinated dibenzo- $p$-dioxin. Microbial community analysis of sediments by $16 \mathrm{~S}$ amplicon sequencing revealed an abundance of lineages from the class Dehalococcoidia (up to $15 \%$ of the community), including the genus Dehalobium (up to 0.5\%). Anaerobic seawater enrichment cultures using perchloroethene as a more amenable growth substrate enriched only the Dehalobium population by more than six-fold. The enrichment culture then proved capable of reductively dechlorinating 2,3,7,8-TCDD to 2,3,7-TCDD and octachlorodibenzo- $p$-dibenzodioxin to hepta and hexa congeners. This work is the first to show microbial reductive dehalogenation of 2,3,7,8-TCDD with a bacterium from outside the Dehalococcoides genus, and one of only a few that demonstrates PCDD/F degradation in a marine environment. 
35

36

37

38

39

40

41

42

43

44

45

46

47

48

49

50

51

52

53

54

55

56

57

58

59

60

61

62

63

64

65

66

67

68

\section{Introduction}

Poly chlorinated dibenzo- $p$-dioxins and furans (PCDD/F) are ubiquitous and persistent organic pollutants. Their ubiquity is due to their production by a number of both natural and anthropogenic processes. The most significant process being combustion of organic matter such as in wildfires and volcanic eruptions ${ }^{1}$. Anthropogenic combustion events such as waste and fuel (wood and coal) burning also contribute to PCDD/F global background concentrations ${ }^{2,3}$. PCDD/F have long been viewed as threat to human and environmental health because they are highly toxic, lipophilic and resistant to metabolism, resulting in bioaccumulation and biomagnification ${ }^{4}$. 2,3,7,8-tetrachlorodibenzo- $p$-dioxin $(2,3,7,8-T C D D)$ is one of the most toxic substances known with an $\mathrm{LD}_{50}$ of 0.6 $\mu \mathrm{g} . \mathrm{kg}^{-1}$ in guinea pigs ${ }^{5,6}$. The PCDD/F toxicity mode is through binding to the aryl hydrocarbon receptor (AHR). The degree of toxicity of each congener correlates with its binding affinity for the AHR ${ }^{7}$. Through this phenomenon toxicological equivalency factors (TEFs) were assigned to the 17 most problematic PCDD/F congeners (i.e. seven PCDDs and $10 \mathrm{PCDF})^{8}$. TEFs range in orders of magnitude difference, the highest being 2,3,7,8-TCDD (TEF = 1) and the lowest being octachlorodibenzo- $p$-dioxin (OCDD, TEF $=0.0003)^{8}$. The toxicity equivalent (TEQ) is the toxicity-weighted mass of each individual congener. In 1998 the World Health Organization (WHO) adopted the TEF approach as a means to quantify PCDDF/F and compare toxicity in environments containing congener mixtures ${ }^{4}$.

Sydney Harbour Estuary includes the waters of the Sydney Harbour, Middle Harbour and the Parramatta River, and is the centerpiece of the city of Sydney, the most populated city in Australia ( $>5$ million people). The estuary has been the site of heavy industrial activity since the early $1800 \mathrm{~s}^{9}$. One notable industry that has left an indelible mark on the estuary was a chemical manufacturing site on Homebush Bay, approximately $15 \mathrm{~km}$ west of the estuaries entrance. Homebush Bay is a $0.8 \mathrm{~km}^{2}$ embayment opening into the Parramatta River. For more than 50 years Union Carbide operated a facility that synthesized pentachlorophenol (PCP, a timber preservative) and the herbicides 2,4-dichloro and 2,4,5-trichlorophenoxyacetic acid (2,4-D and 2,4,5-T ${ }^{10}$. By-products from 
69 these syntheses include: Octa, hepta and hexa congeners of PCDD/F from PCP, and 2,3,7,8-TCDD from 2,4-D and 2,4,5-T5,11. Prior to 1970 PCDD/F waste from the facility was landfilled nearby and contaminated soil was then used to reclaim land from the eastern side of bay, eventuating in massive contamination of the bays sediments ${ }^{10}$.

The most recent PCDD/F concentration data for Sydney Harbour Estuary sediments was reported by Birch et al., in 200712. The analyses revealed that sediments on the eastern side of the bay had a maximum concentration of $6,920,000$ pg.g-1 (4352 pg WHO-TEQ g-1), with more the $50 \%$ of the toxicity being attributed to 2,3,7,8-TCDD ${ }^{12}$. These concentrations placed it among the worlds most PCDD/F contaminated sites, surpassed only by Frierfjorden in the Netherlands, which had a reported maximum PCDD/F concentration of 19,444 pg WHO-TEQ g-1 in 200712. Furthermore, PCDD/F were detected more than 15 $\mathrm{km}$ from Homebush Bay with the same distinct congener profile, revealing that the Homebush Bay is the contaminant source for the entire estuary.

To date no evidence of PCDD/F degradation has been reported for Sydney Harbour sediments. In anaerobic environments such as those found in marine and estuarine sediments, organohalide respiring bacteria (ORB) can reductively dechlorinate aliphatic and aromatic organohalides by using them as respiratory terminal electron acceptors ${ }^{13}$. Obligate and facultative ORB hail from diverse phyla including: Chloroflexi, Firmicutes and Proteobacteria ${ }^{14}$. However, only Dehalococcoides mccartyi strains (Chloroflexi) have been shown to use PCDD/F as terminal electron acceptors (i.e. strains 19515, DCMB516, CBDB117, H1-3-2.001 and KKB3.00318). Strain CBDB1 is the only strain shown to dechlorinate $2,3,7,8-$ TCDD ${ }^{17}$. The genus Dehalococcoides belongs to the class Dehalococcoidia, along with two other obligate organohalide respiring genera (i.e. Dehalogenimonas, Dehalobium $^{19}$ ). While Dehalococcoides strains have been shown to be the sole utilizers of PCDD, Dehalobium phylotypes have been shown to use "dioxin like" polychlorinated biphenyls (PCBs) particularly in estuarine environments ${ }^{20-23}$. Studies on PCDD/F dechlorination in marine environments are rare, and little is 
101 known about the microbial communities associated with PCDD/F in marine and

102 estuarine environments.

103

104 In this study we investigated PCDD/F reductive dechlorination in Sydney

105 Harbour Estuary sediments by comparing absolute and relative PCDD/F

106 concentrations at the source area with nine surrounding bays, and with

107 previously published data ${ }^{12}$. Microbial community analysis of the sediments

108 revealed an abundance of uncultured marine Dehalococcoidia phylotypes, and

109 one cultured phylotype closely aligned with the Dehalobium genus. Attempts to

110 enrich ORB and associated Rdase enzymes with perchloroethene enriched only

111 the Dehalobium phylotype. This enrichment culture was then shown to

112 reductively dechlorinate $2,3,7,8-T C D D$ and OCDD.

113

\section{Materials and methods}

\section{Sediment sampling}

116 Sediment sample cores were collected from nine locations along the Parramatta

117 River. Three samples were take from Homebush Bay and six from surrounding

118 bays spanning a distance of $8.5 \mathrm{Km}$ from the PCDD/F source zone (Site 1) in

119 Homebush Bay to Tarban Creek (Site 9) (Fig. S1, Table S1). The sediments were

120 manually cored in water less the $2 \mathrm{~m}$ in depth by stabbing a $0.5 \mathrm{~m}$ long $\mathrm{x} 0.02 \mathrm{~m}$

121 wide poly carbonate tube attached two a $3 \mathrm{~m}$ aluminum long pole into the

122 sediment. The core samples were immediately capped and sealed with PVC tape.

123 With in 4 hours of sampling the sediments cores were transferred to an

124 anaerobic chamber where there were divided into $25 \mathrm{~cm}$ upper and lower

125 fractions, transferred to glass bottles and sealed with rubber stoppers under an

126 atmosphere of nitrogen.

127

\section{Sample preparation for PCDD/F quantification}

129 Wet sediment was dried overnight at $70^{\circ} \mathrm{C}$, the dried sediment was then ground

130 to a fine powder with a mortar and pestle. $20 \mathrm{~g}$ of sample was then transferred to

131 an automatic solvent extraction (ASE) cartridge along with $50 \mathrm{~g}$ of diatomaceous

132 earth. The sample was spiked with 10 pg.g-1 of an isotopically labelled surrogate

133 compound mixture containing ${ }^{13} \mathrm{C}$ labelled surrogates of the 17 congeners 
134 analyzed (Cambridge Isotope Laboratories Inc. Part \# EDF 5393). The samples

135 were extracted with toluene at $200^{\circ} \mathrm{C}$ and 2000 psi for $10 \mathrm{~min}$ (Dionex ASE 350).

136 The toluene was removed under a stream of nitrogen and the residue was

137 reconstituted in dichloromethane (DCM, $1 \mathrm{ml}$ ) and then applied to a $5 \mathrm{~cm} \times 1.5$

$138 \mathrm{~cm}$ column of Florisil. The PCDD/F were eluted with DCM (50 ml). The DCM was

139 removed under a stream of nitrogen; the PCDD/F residue was reconstituted in a

140 toluene $(0.5 \mathrm{ml})$ and transferred to a $2 \mathrm{ml}$ screw cap glass GC vial.

141

142

PCDD/F analysis and quantification

143 PCDD/F analysis was performed of an Agilent 7890 gas chromatograph (GC)

144 interfaced to an Agilent 7000C triple quadrupole mass spectrometer. The GC was

145 fitted with DB5 (30 m $\times 0.32 \mathrm{~mm}$ (internal diameter) $\times 0.25 \mu \mathrm{m}$ (film thickness)

146 column (Agilent technologies, part \# 123-5062). The carrier gas (He) flow rate

147 was $0.963 \mathrm{ml} \cdot \mathrm{min}^{-1}$. The oven temperature programme was $70^{\circ} \mathrm{C}(1 \mathrm{~min})$ then

148 ramped to $150{ }^{\circ} \mathrm{C}\left(15^{\circ} \mathrm{C} \cdot \mathrm{min}^{-1}\right)$, then ramped to $290^{\circ} \mathrm{C}\left(10^{\circ} \mathrm{C} \cdot \mathrm{min}^{-1}\right)$ and then held

149 at this temperature for 20 mins. The inlet was operated in splitless mode at

$150250^{\circ} \mathrm{C}$. The injection volume was $1 \mu \mathrm{l}$ and the transfer line was maintained at

$151290^{\circ} \mathrm{C}$. The MS was operated in multiple reaction-monitoring mode (MRM). The

152 mass transitions for each congener and analogous isotopically labelled surrogate

153 are provided in Table S2. PCDD/F were quantified by interpolation of a 5-point

154 calibration curve constructed by plotting peak area versus concentration of each

155 congener or surrogate. The concentrations ranged from 0.1 to $1000 \mathrm{ng} \cdot \mathrm{mL}^{-1}$ for

156 each congener and 0.1 to $100 \mathrm{ng} / \mathrm{ml}$ for surrogates (Cambridge Isotope Labs Inc.

157 Part\# EDF-5524) (Table S3). The congener of interest was then multiplied by

158 the recovery ratio of its analogous labelled surrogate.

159

160 The concentration of each of the 17 congeners was converted to pg WHO-TEQ g-1

161 by multiplying it by its TEF (Table S4).

162

163 DNA extraction and 16S rRNA Illumina sequencing

164 DNA was extracted from sediment using a standard phenol chloroform

165 extraction method. To wet sediment (5 g) in a screw cap polypropylene tube was

166 added $6 \mathrm{~mL}$ of lysis buffer (40 mM EDTA, $50 \mathrm{mM}$ Tris- $\mathrm{HCl}, 0.75 \mathrm{M}$ sucrose, $\mathrm{pH}$ 
$1678.0)$ and $200 \mu \mathrm{l}$ of lysozyme (125 mg.ml-1). The tubes were incubated at $37^{\circ} \mathrm{C}$ for

$1681 \mathrm{~h}$ after which $4 \mu \mathrm{l}$ of RNAse A (100 mg.ml-1 $), 5 \mu \mathrm{l}$ of Proteinase K (20 mg.mL $\left.\mathrm{mL}^{-1}\right)$

169 and $200 \mu \mathrm{l}$ of SDS were added to each tube at incubated then incubated at $55^{\circ} \mathrm{C}$

170 for $3 \mathrm{~h}$. The tubes were frozen and then thawed the following day at which time 3

$171 \mathrm{ml}$ of aqueous phase was processed by extraction with phenol-chloroform-

172 isoamyl alcohol. DNA was precipitated with isopropanol, washed twice with ice

173 cold $80 \%$ ethanol and finally dissolved in $30 \mu \mathrm{l}$ of DNA-free molecular grade

174 water. Regions of $16 \mathrm{~S}$ rDNA gene were amplified by PCR from extracted DNA

175 with the Q5 high-fidelity DNA polymerase (New England BioLabs) using the

176 universal primers 926F. 50-TCGTCGGCAGCGTCAGATGTGTATAAGAGACAG -

177 [AAA CTYAAAKGAATTGRCGG]-3?]

178 (5)GTCTCGTGGGCTCGGAGATGTGTATAAGAGACAG -[ACG GGC GGT GTG TRC-

179 3[) targeting bacteria and archaea ${ }^{24}$. The samples were sequenced on an

180 Illumina MiSeq Sequencer (Illumina, USA) using V3 chemistry at the Next

181 Generation Sequencing Facility at Western Sydney University's Hawkesbury

182 Institute for the Environment (Sydney, Australia). 16S rRNA gene amplicon

183 sequences were analyzed with QIIME2-2021.8 utilizing the dada2 pipeline 25,26.

184 Sequencing quality was first visualized with FastQC

185 (www.bioinformatics.babraham.ac.uk) resulting in forwards and reverse reads

186 being trimmed to 220 base pairs. Forward and reverse sequences that passed the

187 default quality control were merged and non-overlapping sequences were

188 discarded. Chimeras were analyzed and removed via the consensus method

189 within the dada2 pipeline. Remaining sequences had taxonomy assigned with the

190 RDP classifier ${ }^{27}$ using the Silva 132 Qiime release database.

191

\section{Perchloroethene enrichment culture preparation}

193 To enrich ORB in the harbour sediments, sediments from the most contaminated

194 Site (Site 1, Lower $50 \mathrm{~cm}$, Figure S1A) were used as the inoculum source. The

195 PCE enrichment was prepared using filter-sterilised seawater amended with

196 mineral salts (g/L) $\left[\mathrm{NaCl}(1.0), \mathrm{MgCl}_{2} \cdot \mathrm{H}_{2} \mathrm{O}(0.5), \mathrm{KH}_{2} \mathrm{PO}_{4}(0.2), \mathrm{NH}_{4} \mathrm{Cl}\right.$ (0.3), $\mathrm{KCl}$

197 (0.3), $\left.\mathrm{CaCl}_{2} \cdot 2 \mathrm{H}_{2} \mathrm{O}(0.015)\right]$, trace elements ${ }^{28}$ and sodium acetate (30 mM). After

198 deoxygenating by nitrogen sparging for $45 \mathrm{~min}$, medium (100 ml) was dispensed

199 into a $120 \mathrm{ml}$ serum flasks containing sediment $(1 \mathrm{~g})$. The flasks were sealed with 
200 Teflon coated rubber septa and aluminum crimps. The headspace was flushed

201 with $\mathrm{N}_{2} / \mathrm{CO}_{2}$ (4:1) for 3 min. The medium was supplied with $100 \mathrm{x}$ vitamin

202 solution $29,10 \mathrm{mM} \mathrm{NaHCO}_{3}$, and $\mathrm{Na}_{2} \mathrm{~S}(0.2 \mathrm{mM})$. The cultures were amended with

203 neat PCE $(0.2 \mathrm{mM})$ using a $10 \mu \mathrm{l}$ glass syringe. Hydrogen gas ( 0.5 bar) was

204 supplied as an electron donor. All enrichment cultures were incubated statically

205 at $30^{\circ} \mathrm{C}$ in the dark.

206

207 Chlorinated ethene quantification

208 Culture headspace gas $(100 \mu \mathrm{l})$ was withdraw via the septum with a gas tight 209 syringe and manually injected into an Agilent 7890A GC equipped with a flame 210 ionization detector (FID) and a GS-Q capillary column (30 $\mathrm{m} \times 0.32 \mu \mathrm{m} \mathrm{J} \& \mathrm{~W}$ 211 Scientific). The temperature of the split/splitless inlet and detector were set to $212250 \square$. The helium carrier gas flow rate was $3 \mathrm{ml} / \mathrm{min}$. The oven program was 213 initially held at $150 \square$, increased to $250 \square$ at a rate of $30 \square$ per min and held for 2 $214 \mathrm{~min}$. The inlet had a split ratio of 1:10.

\section{Quantitative polymerase chain reaction (qPCR) procedure}

217 The qPCR mixtures contained $2 \mu$ of DNA template, $5 \mu$ l of $2 \times$ SsoFastTM 218 EvaGreen Supermix, $0.1 \mu \mathrm{l}$ each of forward and reverse primers, $0.1 \mu \mathrm{l}$ of Bovine 219 Serum Albumin (BSA) solution and $2.7 \mu \mathrm{l}$ of DNA-free Molecular grade water. 220 Universal bacterial primers Eub1048F (5'-GTGSTGCAYGGYTGTCGTCA-3') and 221 Eub1195R (5'-ACGTCRTCCMCACCTTCCTC-3') were used to quantify the total 222 bacteria concentration ${ }^{30}$. Thermo cycling comprised of $95^{\circ} \mathrm{C}$ for $3 \mathrm{~min}$, followed 223 by 40 cycles of denaturation at $95^{\circ} \mathrm{C}$ for 20 seconds and annealing at $62^{\circ} \mathrm{C}$ for 50 224 seconds. Following amplification, melting curve analysis was conducted with 225 increments at $0.5^{\circ} \mathrm{C}$ per 10 seconds from 60 to $99^{\circ} \mathrm{C}$. Dehalococcoides primers 226 Dehalo505F (5'-GGCGTAAAGTGAGCGTAG-3') and Dehalo686R (5'227 GACAACCTAGAAAACCGC-3') were used to quantify Dehalococcoides $\mathrm{spp}^{31}$. 228 Thermo cycling comprised of $98^{\circ} \mathrm{C}$ for $3 \mathrm{~min}$, followed by 45 cycles of 229 denaturation at $95^{\circ} \mathrm{C}$ for 30 seconds and annealing at $58^{\circ} \mathrm{C}$ for 50 seconds. 230 Following amplification, melting curve analysis was conducted with increments 231 at $0.5^{\circ} \mathrm{C}$ per 5 seconds from 55 to $95^{\circ} \mathrm{C}$. 


\section{Sediment microcosms on TCDD and OCDD transformation}

234 TCDD and OCDD were adsorbed onto dried sediments for use as stocks. TCDD

235 and OCDD were dissolved in hexane $(50 \mathrm{ml})$ and mixed with $20 \mathrm{~g}$ of dried

236 sediment with the lowest PCDD/F concentration (Site 9). The hexane was

237 evaporated under a stream of nitrogen resulting in 2,3,7,8-TCDD and OCDD

238 fortified sediment with $3.0 \mu \mathrm{g} \cdot \mathrm{g}^{-1}-\mathrm{dw}$ and $54 \mu \mathrm{g} \cdot \mathrm{g}^{-1}-\mathrm{dw}$, respectively.

239

240 To test for 2,3,7,8-TCDD and OCDD biotransformation, PCE/sediment 241 enrichment microcosms were purged with $\mathrm{N}_{2}$ for $30 \mathrm{~min}$ to remove all 242 chloroethenes, and 10\% (v/v) inoculated into an anaerobic artificial seawater 243 medium $(2 \% \mathrm{NaCl})$ and spiked with $1 \mathrm{~g}$ of 2,3,7,8-TCDD or OCDD stock dry244 sediments. $\mathrm{H}_{2}$ (0.5 bar) and sodium acetate $(30 \mathrm{mM})$ were supplied as electron 245 donor and organic carbon source. The $\mathrm{H}_{2}$ pressure was returned to 0.5 bar every 246 month. All enrichment cultures were incubated statistically at $30^{\circ} \mathrm{C}$ in the dark.

\section{Statistical analysis}

249 Statistical analysis was performed using Prism 9 for MacOS (version 9.1.2).

250 Student's t-test were two-tallied and unpaired and equal variance was not 251 assumed (unless otherwise stated). Principal component analysis was performed 252 as previously described for PCDD/F in Sydney Harbour Estuary ${ }^{12}$. Absolute 253 PCDD/F concentrations were normalized by dividing by the most abundant 254 congener (either OCDD of OCDF, note Dioxins and furans were treated 255 separately). PCA was then performed on the $\log _{10}$ values of the normalized 256 congener concentrations.

257

\section{Results and discussion}

259 PCDD/F concentrations in Sydney Harbour Estuary sediments

260 The PCDD/F source area adjacent to the former on the eastern side of Homebush 261 Bay was the most contaminated with $1971 \times 10^{3}$ and $4556 \times 10^{3}$ pg.g $^{-1}$ (2026 and

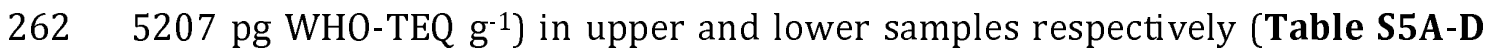
263 and Fig. S2 A). The two other Homebush Bay samples (Sites 2 and 3) had similar 264 concentrations to each other in both upper and lower fractions, with the mean 265 being $540 \pm 69$ pg WHO-TEQ g-1 $(n=4)$. (Fig. S2B). A comparison of mean upper 
266 and lower concentrations in the surrounding bays was made with Homebush

267 Bay Sites 2 and 3 (the source was excluded). The comparison showed that there

268 was no significant difference in the total PCDD/F concentrations up to $4.5 \mathrm{~km}$

269 away $(p>0.05)$. However, at $8 \mathrm{~km}$ the PCDD/F concentrations were significantly

270 lower (Fig. S2B).

271

272 In all 18 samples the PCDDs where in greater abundance than PCDFs. When

273 averaged across all sampling sites PCDDs were $18.2 \pm 4.0$ and $21.3 \pm 11.5$ times

274 greater than PCDFs in upper and lower sediments respectively (Table S5A \& B).

275 The perchlorinated congeners OCDD or OCDF were consistently the most

276 abundant within their chemical class (i.e. PCDD or PCDF) across all nine locations

277 at both depths. OCDD accounted for $96.3 \pm 8.4 \%(n=18)$ of the PCDDs and OCDF

278 accounted for $94.3 \pm 7.7 \%(\mathrm{n}=18)$ of PCDFs. 2,3,7,8-TCDD accounted for $0.02 \pm$

$2790.006 \%$ of the PCDD/F mass and $15.9 \pm 8.4 \%(n=18)$ of the toxicity (Table S5

280 A-D).

281

282 Principal component analysis of PCDD/F congener profiles

283 Principal component analysis (PCA) of normalized congener profiles previously

284 showed a tight clustering of samples from Homebush Bay with surrounding 285 areas $^{12}$. From this correlation the authors concluded that the PCDD/F 286 contamination at Homebush Bay was the source of contamination for 287 surrounding areas of the estuary extending $5 \mathrm{~km}$ to the west and $15 \mathrm{~km}$ to the 288 east of the source. Similarly, we performed PCA on $\log _{10}$ of normalized congener 289 concentrations at all sample locations. Normalized congener concentrations 290 were achieved by dividing by the most abundant congener (either OCDD of 291 OCDF, note Dioxins and furans were treated separately). The Homebush Bay 292 congener profiles from 2007 were treated in the same way and included for 293 comparison (Fig 1A). As expected the Homebush Bay congener profiles from 2942007 clustered together. In contrast the tight clustering of samples was no longer 295 evident in congener profiles determined in the present study. The source area is 296 clearly different to Sites 2 and 3 in Homebush Bay (that cluster together) and 297 surrounding bays (Sites 4-9). These results suggest that significant changes in 
298 the congener profiles have occurred in the 14 years between studies particularly in Homebush Bay.

Figure 1. Panel A. PCA plot of congener profiles at Homebush Bay and surrounding Bays. Each congener concentration was normalized to the total congener concentration and then transformed ( $\left.\log _{10}\right)$. Data from 2007 were included for comparison (circled in blue). Homebush Bay 2017 samples (circled in red), 2017 Surrounding Bays (circled in green), 2017 source zone (circled in pink). Panel B. $t$-test comparison of mean (upper and lower) normalized congener concentrations at the source zone with the other eight locations. (dark blue indicates a $p$-value $>0.1$ ). Panels $\mathbf{C}$ - G shows the normalized congener concentrations that are significantly different to those at the source

To elucidate the significant differences in congener profiles at the source compared to other sites, a pair wise comparison was made in the relative abundance of each congener at the source with the corresponding congener at each of the sampling sites (Fig. 1B). The pairwise $t$-test comparisons revealed significant $(p<0.05$ ) differences in PCDFs, 1,2,3,4,6,7,8-HepCDF, 1,2,3,7,8,9- and 2,3,4,6,7-HexCDF (Fig 2C). A closer inspection of these congeners revealed a lower relative abundance of HepCDF, accompanied by elevated relative abundance of 2,3,4,6,7,8-hexaCDF at the source compared with the other locations, suggesting that reductive dechlorination of the hepta congener has occurred at the source (Fig 2B \& C). The proportion of 1,2,3,7,8,9-hexaCDF was also significantly lower at the source relative to all other locations (Fig. 2D). However, there was no significance difference in the proportion of penta congeners, suggesting that $1,2,3,7,8,9$-hexaCDF was transformed to a penta congener that was not amongst the suite of compounds analyzed. Regarding PCDDs $1,2,3,4,7,8-H e x C D D$ was significantly higher in relative abundance at the source than at all sites, a potential precursor to $1,2,3,4,7,8-H e x C D D$, i.e. $1,2,3,4,6,7,8$-heptaCDD was not significantly different in relative abundance across the nine sites (Fig 2F).

\section{Comparison of Homebush Bay congener profiles with historical data}

\section{indicates 2,3,7,8-TCDD degradation}

333 from similar areas to those in $2007^{12}$ (Fig S3). Samples were grouped into those

334 at the source area and those outside the source area for comparison of congener 
335 TEQs and profiles (Fig $\mathbf{3 A} \& \mathbf{C}$ ). In both cases the most significant congener in 3362007 in terms of toxicity was 2,3,7,8-TCDD (Fig. 3A\&C). However in the present 337 study the 2,3,7,8-TCDD was significantly lower than historical levels both inside 338 ( $p=0.013)$ and outside $(p=0.015)$ the source area. The reduced abundance of 339 2,3,7,8-TCDD was also evident in the normalized congener TEQ profile 340 demonstrating that 2,37,8-TCDD had diminished in abundance relative to all 341 other congeners as well as relative to historical values (Fig 3B \& D).

342

343

344

345

346

347

348

349

350

351

352

353

354

355

356

357

358

359

360

361

362

363

364

365

366

367

368

369

Figure 2. Homebush Bay PCDD/F congener concentrations and normalized concentrations in 2007 (historical ${ }^{12}$ ) and 2017 (present study). Panel A-B

Source area on the eastern side the bay. Panels C-D sample locations outside the source area (Precise locations are shown in Figure S3).

Analysis of the sediment extracts for 2,7,8-triCDD, 2,7-/2,8-DiCDD, chloroCDD revealed all but 2,7,8-TriCDD to be present suggesting that reductive dechlorination could be a mechanism of 2,3,7,8-TCDD transformation (Fig S4). However, as there is no prior data for lesser-chlorinated PCDDs in the harbour sediments it is not known if they were part of the initial pollutant mix or if they are evidence of in situ 2,3,7,8-TCDD reductive dechlorination. Nevertheless, this study provides baseline data for future comparison.

\section{$16 S$ amplicon sequencing reveals potential PCDD/F respiring bacteria}

The microbial community analysis in this study was performed primarily to identify ORB that have the potential to reductively dechlorinate PCDDs, and specifically 2,3,7,8-TCDD (i.e. Dehalococcoides mccartyi strains). DNA extracted directly from the sediment samples was analyzed by $16 \mathrm{~S}$ rRNA gene amplicon sequencing. The number of sequences retrieved ranged between 49158 to 115942 (mean $=77216 \pm 22914(\mathrm{n}=18)$. The community consisted of 15 phyla with a relative abundance above $0.5 \%$ of which 12 were represented in all samples (i.e. the microbial core community) (Fig 3A). The core community represented $>94 \%$ of identified phyla; the non-core community ranged between 0.7 and $6 \%$.

Chloroflexi was the most abundant phyla accounting for $28.1 \pm 11.3 \%$ of the community across all samples (n=18) (Fig 3B). The class Dehalococcoidia in the 
370 Chloroflexi phylum accounted for $10.9 \pm 9.30 \%$ of the total community second to 371 Anaerolineae (16.8 5.37\%). Previously, 16S rRNA phylogenetic analysis of 372 orders within Dehalococcoidia have shown them to assemble in three clusters 373 (i.e. Marine I, Marine II, and Terrestrial I) ${ }^{32}$. All cultivated phylotypes belong to 374 Terrestrial Cluster I, and are characterised by their ability to conserve energy via 375 respiration of organohalides ${ }^{32}$. The physiological role of Dehalococcoidia in 376 marine sediments is unclear primarily due to a lack of cultivated phylotypes. 377 However, analysis of metagenome and single cell assembled genomes (MAGS and 378 SAGS) of marine Dehalococcoidia have revealed a general absence of reductive 379 dehalogenase genes suggesting that they conserve energy though means other 380 than organohalide respiration ${ }^{33}, 34$. In the present study Dehalococcoidia 381 affiliates were denominated as uncultivated lineages with the order GIF9 382 (Marine Cluster II) being the most abundant across the 18 samples (38.2 $\pm 15.8 \%$ 383 of Dehalococcoidia) (Fig 3C). GIF9 has been shown to possess only a partial 384 reductive dehalogenase gene and therefore it seems unlikely that it is 385 participating in PCDD/F dechlorination ${ }^{32}$. Dehalobium in the Dehalococcoidales 386 order was the only cultivated lineage identified at the genus level and accounted 387 for a small proportion of the Dehalococcoidia class $(0.33 \pm 0.51 \%(n=18))$.

388 Dehalobium species have been shown to use PCE and PCBs as terminal electron 389 acceptors in marine environments ${ }^{20-22}$. However, there are no reports of 390 Dehalobium species dechlorinating PCDD/F.

Figure 3. Panel A. Relative abundance of the core bacterial phyla in Sydney Harbour sediments at the level of Phylum. Panel B. Class within the Chloroflexi phyla. Panel C. Order within the Dehalococcoidia class (C).

\section{Enrichment of Dehalobium spp. using PCE as electron acceptor}

397 Given the lack of clarity regarding ORB within the sediments it was decided to 398 attempt to enrich the ORB population using PCE. PCE was chosen because 399 Dehalococcoides mccartyi strain CBDB1 is capable of using both PCE and 2,3,7,8400 TCDD as its terminal electron acceptor ${ }^{17}$, 35. However, PCE with an aqueous 401 solubility of $150 \mathrm{mg} . \mathrm{L}^{-1}$ has far greater bioavailability than 2,3,7,8-TCDD with an 402 aqueous solubility of $0.2 \mu \mathrm{g} . \mathrm{L}^{-1(36)}$. 
404 The enrichment cultures were prepared with sediment from source zone (lower 405 fraction) with filter-sterilized seawater to replicate in situ conditions. The first 406 PCE (0.2 mM) pulse was dechlorinated to TCE $(0.10 \mathrm{mM})$ and cis-DCE (0.06 mM). 407 Additional PCE (0.2 to $0.4 \mathrm{mM}$ ) was subsequently supplied. After 85 days the 408 best performing microcosm had dechlorinated $2.60 \mathrm{mM}$ PCE to TCE (1.41 mM) 409 and cis- and trans-DCE (0.74 and $0.44 \mathrm{mM}$, respectively) (Fig 4A). No further 410 dechlorination to vinyl chloride or ethene was observed. Sulfate reduction was 411 also observed and was completely depleted from the seawater medium (data not 412 shown). Neither PCE or sulfate reduction occurred in sterilized controls.

The PCE-enrichment culture was analyzed by Illumina 16S rRNA gene amplicon 415 sequencing at day 0 and 90 (Fig 4B). After 90 days, the only ORB was identified 416 in the community was Dehalobium from the Dehalococcoidia class, (3.6\% of the 417 community) (Fig 4B). No other Dehalococcoidia lineages that were present at 418 day zero were detectable at day 90. The production of both cis- and trans-DCE in 419 a ratio of 1.5 is consistent with previous reports for Dehalobium lineages when 420 respiring $\mathrm{PCE}^{37}$. Blastn alignment of the 266 base-pair representative sequences 421 aligned most closely with uncultured Dehalococcoidia VLD-1 (98.95\% identity), 422 which is closely related to Dehalobium chlorocoercia DF-1 (Fig 4C). A large 423 increase in the relative abundance of sulfate reducing bacteria including 424 Desulfovibrio (52\%) and the Desulfobulbaceae family (15\%) was observed. Their 425 proliferation is consistent with the observed removal of sulfate from the 426 seawater medium.

428 Figure 4. Panel A. PCE dechlorination by a sediment enrichment culture in 429 seawater supplied with hydrogen and acetate as electron donor and carbon 430 source respectively. Panel B 16S rRNA community analysis of the PCE 431 enrichment culture at time zero and day 90. Panel C Maximum likely-hood 432 Phylogenetic tree of Dehalococcoidia partial 16S rRNA gene sequences acquired 433 in this study from Sydney Harbour Estuary sediments (labelled SH) relative to 434 cultivated phylotypes in the Dehalococcoidia class. Sequence alignment, 435 modeling and construction were performed with MEGA (version 10.2.6). 
436 Numbers next to the nodes represent the percentage of branch support based on

437 bootstrap re-sampling (500x).

438

439 TCDD and OCDD dechlorinating activity in ORB enrichment culture

440 The PCE enrichment culture was applied in anaerobic cultures containing

$4412,3,7,8-T C D D$ and OCDD as electron acceptors (i.e. the congener with the highest

442 TEQ and the most abundant by mass respectively) and hydrogen as the electron

443 donor. A separate set of cultures was prepared that included the biosurfactant

444 lecithin, that has been shown to increase the bioavailability of lipophilic organic

445 pollutants such as PCDD/F and $\mathrm{PCBs}^{38,39}$.

446 The enrichment cultures resulted in the reductive dechlorination of both

447 congeners after a lag of five-months. In 2,3,7,8-TCDD amended microcosms,

448 2,3,7-TriCDD was detectable in the lecithin treatments after 5-months, but not in

449 the lecithin free control. After 14-months 2,3,7,-TriCDD was detected in both

450 lecithin and lecithin free treatments with no statistical difference between the

451 two (Fig 5A). After 28 months of observation there was more 2,3,7-TriCDD

452 produced in the lecithin amended microcosms $\left(0.49 \pm 0.3\right.$ nmoles.g $^{-1}$ vs. $0.10 \pm$

4530.07 nmoles.g ${ }^{-1}(p=0.093)$. The quantity of 2,3,7-TriCDD produced represented

$4545.4 \pm 1.0 \%$ and $1.1 \pm 0.77$ mole $\%$ of the initial amount of $2,3,7,8$-TCDD added to

455 the lecithin and lecithin free microcosms respectively.

456

457

OCDD was dechlorinated to 1,2,3,4,6,7,8-HepCDD and 1,2,3,4,7,8-HexCDD (Fig.

458 5B). HepCDD was detectable after five months where lecithin was employed but

459 not in the lecithin free control. After 28 months of observation the sum of

460 dechlorination products in lecithin-amended microcosms $\left(24.1 \pm 10.1 \mathrm{nmol.g}{ }^{-1}\right)$

461 was significantly higher at the $90 \%$ confidence interval than those without

462 lecithin $\left(10.7 \pm 1.59\right.$ nmol.g $\left.{ }^{-1}\right)(p=0.089)$. No dechlorination was observed in

463 sterile controls, suggesting that the transformation was dependent on microbial

464 activity.

465

466 16S rRNA sequencing of DNA from the cultures after 14 months showed that no

467 other member of Dehalococcoidia class besides Dehalobium were detectable

468 during 2,3,7,8-TCDD and OCDD dechlorination. However, during OCDD 
469 dechlorination the Dehalobium population diminished significantly to below 470 detection in four of six microcosms, whereas in 2,3,7,8-TCDD microcosm the 471 Dehalobium population ranged from $0.33-4.59 \%$ of the microbial population 472 (Fig 5C). This suggests that OCDD dechlorination may not be linked to 473 Dehalobium. The mechanism of dechlorination of OCDD dechlorination could be 474 an abiotic process via reduced cobalamins in the medium. A previous study has 475 shown that reduced cyanocobalamin can catalyze the reductive dechlorination of 476 OCDD to TCDD but not beyond ${ }^{40}$.

Cultures amended with lecithin had increased relative abundance of 479 Clostridiales, known to ferment a range of organic compounds under anaerobic 480 conditions ${ }^{41}$. The relative abundance of Clostridiales in all lecithin-amended cultures accounted for $70 \pm 6.6 \%(n=6)$ of the microbial population compared with $39.4 \pm 5.5 \%(n=6)$ of the population in unamended cultures. This finding suggests that lecithin is being degraded by members of the Clostridiales and hence is utility as long-term biosurfactant to solubilize PCDD/F is doubtful.

Figure 5. The production of 2,3,7-TriCDD from 2,3,7,8-TCDD (Panel A) and 1,2,3,4,6,7,8-HepCDD and 1,2,3,4,7,8-HexCDD from OCDD (Panel B) in anaerobic seawater +/- lecithin microcosms inoculated with the PCE/Dehalobium enrichment culture. Error bars represent standard deviation $(n=3)$. Panel C $16 S$ rRNA community analysis of the 2,3,7,8-TCDD and OCDD microcosms inoculated with PCE/Dehalobium enrichment culture after 14 months of incubation.

\section{Conclusion}

494 In the study we have insight into the natural attenuation of PCDD/F congeners in 495 the sediments in Sydney Harbour Estuary. The most significant transformation 496 observed was a 50\% decrease in the most toxic and concerning congener 2,3,7,8497 TCDD accompanied by the presence of lesser-chlorinated dioxins in the 498 sediments. Taken together these findings suggest that reductive dehalogenation 499 is at least one mechanism by which 2,3,7,8-TCDD is being detoxified. Microbial 500 community analysis of the sediments revealed an abundance of lineages from the 501 Dehalococcoidia class. However, the majority of these lineages were associated 502 with physiologically uncharacterized marine phylotypes whose ability to use 503 organohalides as terminal electron acceptor is in despute. Nevertheless an ORB 
504 from Dehalococcoidia was enriched using PCE as the electron acceptor that 505 phylogenetically aligned with the Dehalobium genus. The Dehalobium 506 enrichment culture was then shown to reductively dechlorinate 2,3,7,8-TCDD 507 and OCDD. Collectively, these findings show promise for the bioremediation of 508 marine sediments contaminated with PCDD/F. Future work will entail defining 509 the repertoire of PCDD/F congeners and other organohalides that this culture 510 can reductively dechlorinate and genetically and functionally characterizing the 511 reductive dehalogenase(s) that catalyze these reactions. PCR primers based 512 RDase gene sequences coupled with the 16S rRNA phylogenetic marker genes 513 will be valuable diagnostic tool available to remediation practitioners to 514 determine the in situ capability for PCDD/F attenuation. 


\section{References}

1. Zook, D. R., et al., Environmental sources, distribution, and fate of polychlorinated dibenzodioxins, dibenzofurans, and related organochlorines. Dioxins and health 1994, 79-113.

2. Thomas, V. M., et al., An estimation of dioxin emissions in the United States. Toxicological \& Environmental Chemistry 1995, 50, (1-4), 1-37.

3. Lei, R., et al., Global status of dioxin emission and China's role in reducing the emission. J. Haz. Materials 2021, 126265.

4. Van den Berg, M., et al., The 2005 World Health Organization reevaluation of human and mammalian toxic equivalency factors for dioxins and dioxin-like compounds. Toxicological sciences 2006, 93, (2), 223-241.

5. Hites, R. A., Dioxins: an overview and history. Environ. Sci. Technol. 2011, 45, (1), 16-20.

6. Schwetz, B., et al., Toxicology of chlorinated dibenzo-p-dioxins. Environ. Health Perspectives 1973, 5, 87-99.

7. Safe, S. H., Comparative toxicology and mechanism of action of polychlorinated dibenzo-p-dioxins and dibenzofurans. Annual review of pharmacology and toxicology 1986, 26, (1), 371-399.

8. Safe, S. H., Development validation and problems with the toxic equivalency factor approach for risk assessment of dioxins and related compounds. Journal of animal science 1998, 76, (1), 134-141.

9. Birch, G., A short geological and environmental history. In Sydney University Press: 2006.

10. Montoya, D., Pollution in Sydney Harbour: sewage, toxic chemicals and microplastics. NSW Parliamentary Research Service: 2015.

11. Verbrugge, L. A., et al., Pentachlorophenol, polychlorinated dibenzo-p-dioxins and polychlorinated dibenzo furans in surface soil surrounding pentachlorophenol-treated utility poles on the Kenai National Wildlife Refuge, Alaska USA. Environ. Sci. Pollution Res. 2018, 25, (19), 19187-19195.

12. Birch, G., et al., The source and distribution of polychlorinated dibenzo-p-dioxin and polychlorinated dibenzofurans in sediments of Port Jackson, Australia. Marine pollution bulletin 2007, 54, (3), 295-308.

13. Jugder, B.-E., et al., Organohalide respiring bacteria and reductive dehalogenases: key tools in organohalide bioremediation. Frontiers in microbiology 2016, 7, 249.

14. Jugder, B.-E., et al., Reductive dehalogenases come of age in biological destruction of organohalides. Trends Biotechnol. 2015, 33, (10), 595-610.

15. Fennell, D. E., et al., Dehalococcoides ethenogenes strain 195 reductively dechlorinates diverse chlorinated aromatic pollutants. Environ. Sci. Technol. 2004, 38, (7), 2075-2081.

16. Pöritz, M., et al., Dehalococcoides mccartyi strain DCMB5 respires a broad spectrum of chlorinated aromatic compounds. Appl. Environ. Microbiol. 2015, 81, (2), 587-596.

17. Bunge, M., et al., Reductive dehalogenation of chlorinated dioxins by an anaerobic bacterium. Nature 2003, 421, (6921), 357-360.

18. Dam, H. T., et al., Reconstructed genomes of novel Dehalococcoides mecartyi strains from 1, 2, 3, 4-tetrachlorodibenzo-p-dioxin-dechlorinating enrichment cultures 
reveal divergent reductive dehalogenase gene profiles. FEMS Microbiol. Ecol. 2017, 93, (12), fix151.

19. Yang, Y., et al., Roles of Organohalide-Respiring Dehalococcoidia in Carbon Cycling. Msystems 2020, 5, (3), e00757-19.

20. Fagervold, S. K., et al., Microbial reductive dechlorination of Aroclor 1260 in Baltimore harbor sediment microcosms is catalyzed by three phylotypes within the phylum Chloroflexi. Appl. Environ. Microbiol. 2007, 73, (9), 3009-3018.

21. Cutter, L. A., et al., Identification of a microorganism that links its growth to the reductive dechlorination of 2, 3, 5, 6 - chlorobiphenyl. Environ. Microbiol. 2001, 3, (11), 699-709.

22. May, H. D., et al., Dehalorespiration with polychlorinated biphenyls by an anaerobic ultramicrobacterium. Appl. Environ. Microbiol. 2008, 74, (7), 2089-2094.

23. $\mathrm{Wu}, \mathrm{Q}$., et al., Identification of a bacterium that specifically catalyzes the reductive dechlorination of polychlorinated biphenyls with doubly flanked chlorines. Appl. Environ. Microbiol. 2002, 68, (2), 807-812.

24. Engelbrektson, A., et al., Experimental factors affecting PCR-based estimates of microbial species richness and evenness. The ISME journal 2010, 4, (5), 642-647.

25. Caporaso, J., et al., Correspondence QIIME allows analysis of high-throughput community sequencing data intensity normalization improves color calling in SOLiD sequencing. Nat Publ Gr [Internet] 7 (5): 335-336. In 2010.

26. Callahan, B. J., et al., DADA2: high-resolution sample inference from Illumina amplicon data. Nat. Methods 2016, 13, (7), 581-583.

27. Wang, Q., et al., Naive Bayesian classifier for rapid assignment of rRNA sequences into the new bacterial taxonomy. Appl. Environ. Microbiol. 2007, 73, (16), 5261-5267.

28. Löffler, F. E., et al., Enrichment, cultivation, and detection of reductively dechlorinating bacteria. Methods Enzymol. 2005, 397, 77-111.

29. Wolin, E., et al., Formation of methane by bacterial extracts. J. Biol. Chem. 1963, 238, (8), 2882-2886.

30. Horz, H., et al., Evaluation of universal probes and primer sets for assessing total bacterial load in clinical samples: general implications and practical use in endodontic antimicrobial therapy. Journal of clinical microbiology 2005, 43, (10), 5332-5337.

31. Behrens, S., et al., Monitoring abundance and expression of "Dehalococcoides" species chloroethene-reductive dehalogenases in a tetrachloroethene-dechlorinating flow column. Appl. Environ. Microbiol. 2008, 74, (18), 5695-5703.

32. Yang, Y., et al., Genomic characteristics distinguish geographically distributed Dehalococcoidia. Frontiers in microbiology 2020, 11, 2133.

33. Wasmund, K., et al., Single-cell genome and group-specific dsrAB sequencing implicate marine members of the class Dehalococcoidia (phylum Chloroflexi) in sulfur cycling. MBio 2016, 7, (3), e00266-16.

34. Kaster, A.-K., et al., Single cell genomic study of Dehalococcoidetes species from deep-sea sediments of the Peruvian Margin. The ISME journal 2014, 8, (9), 1831-1842.

35. Marco-Urrea, E., et al., Transformation and carbon isotope fractionation of tetraand trichloroethene to trans-dichloroethene by Dehalococcoides sp. strain CBDB1. Environ. Sci. Technol. 2011, 45, (4), 1555-1562. 
36. Shiu, W. Y., et al., Physical-chemical properties of chlorinated dibenzo-p-dioxins. Environ. Sci. Technol. 1988, 22, (6), 651-658.

37. Kittelmann, S., et al., Novel uncultured Chloroflexi dechlorinate perchloroethene to trans - dichloroethene in tidal flat sediments. Environ. Microbiol. 2008, 10, (6), 1557 1570 .

38. Fava, F., et al., Soya lecithin effects on the aerobic biodegradation of polychlorinated biphenyls in an artificially contaminated soil. Biotechnol. Bioeng. 2001, 72, (2), 177-184.

39. Soeder, C., et al., Influence of phytogenic surfactants (quillaya saponin and soya lecithin) on bio-elimination of phenanthrene and fluoranthene by three bacteria. Appl. Microbiol. Biotechnol. 1996, 44, (5), 654-659.

40. Adriaens, P., et al., Dechlorination of PCDD/F by organic and inorganic electron transfer molecules in reduced environments. Chemosphere 1996, 32, (3), 433-441.

41. Boutard, M., et al., Functional diversity of carbohydrate-active enzymes enabling a bacterium to ferment plant biomass. PLoS Gen. 2014, 10, (11), e1004773. 

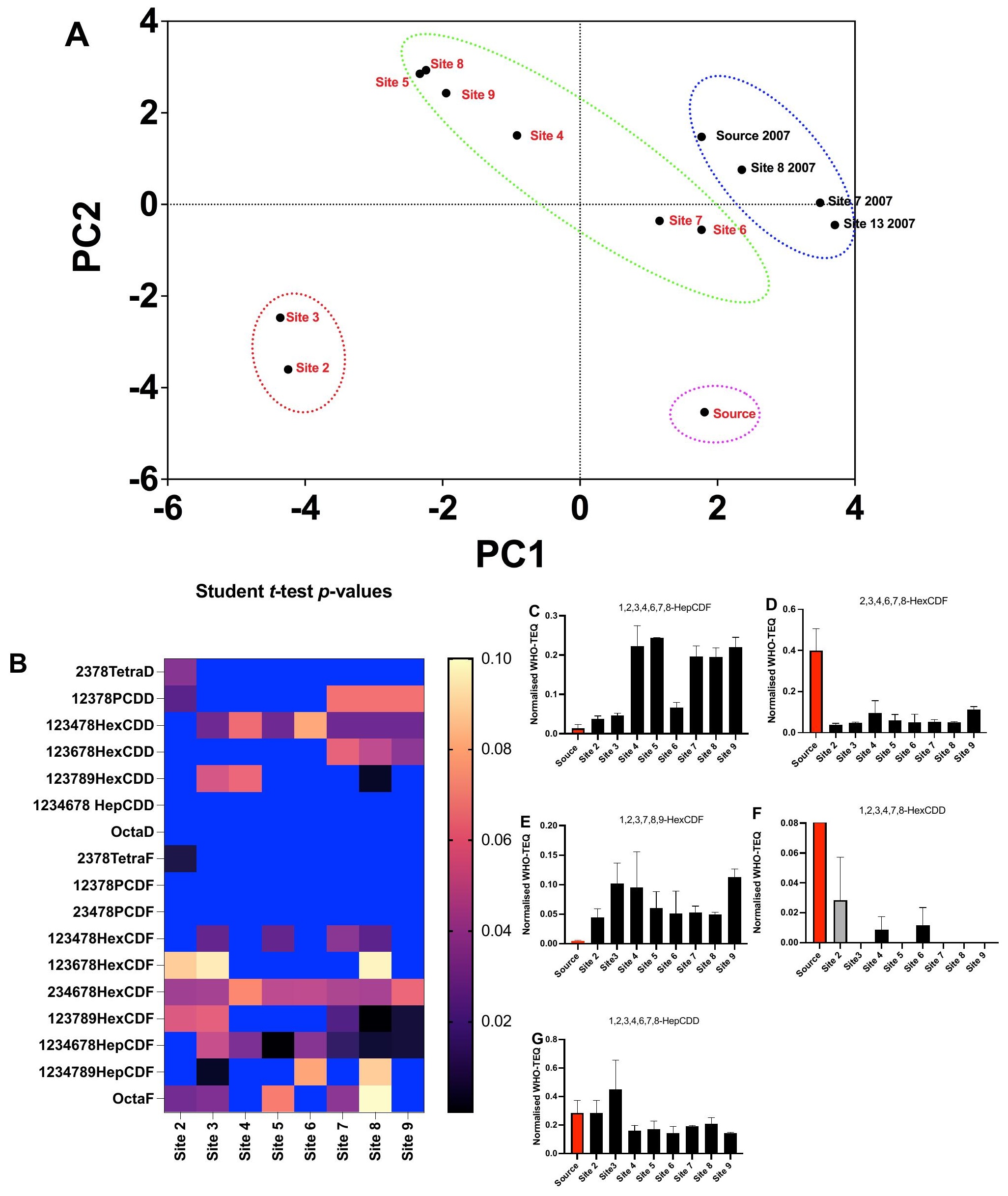

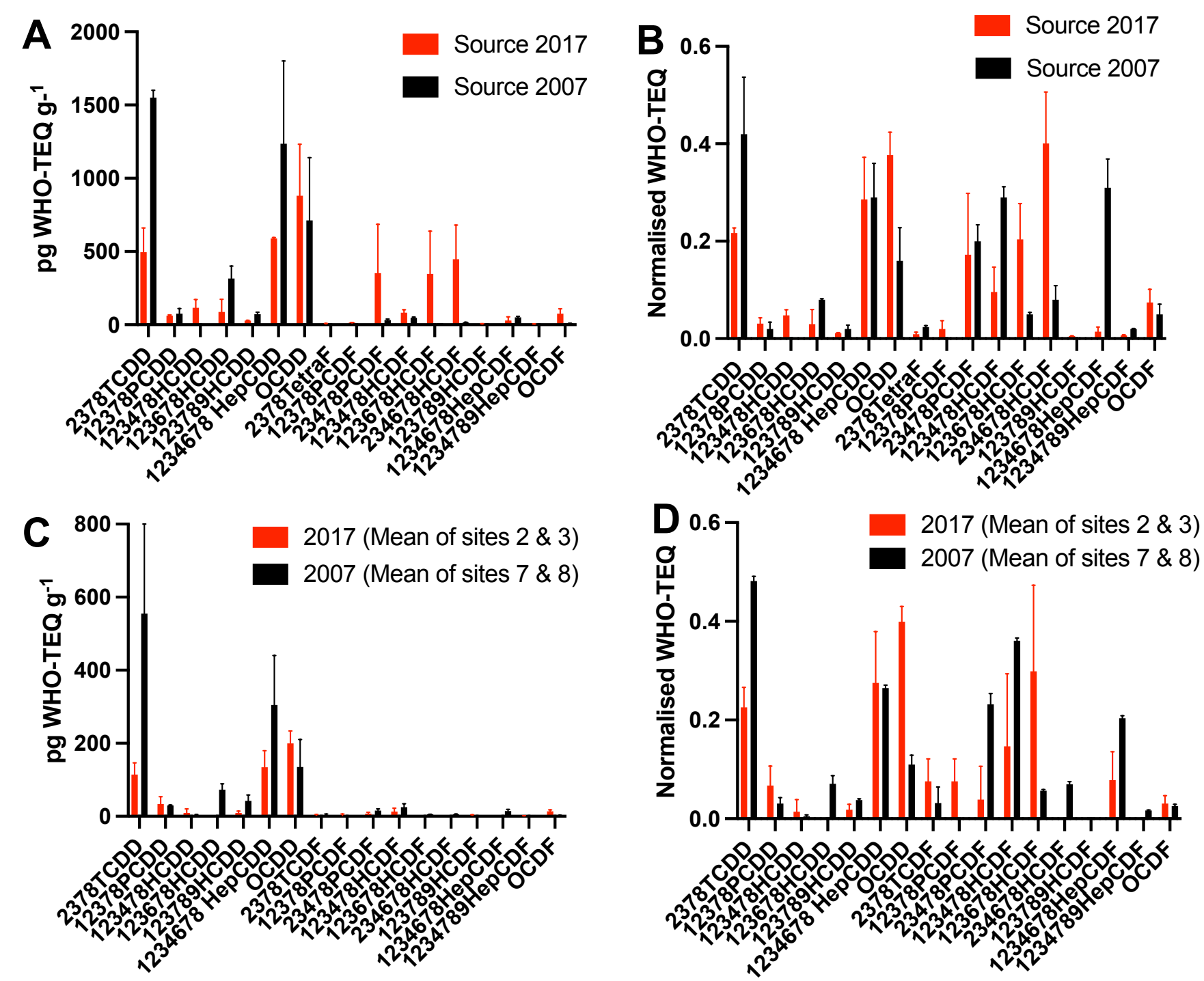


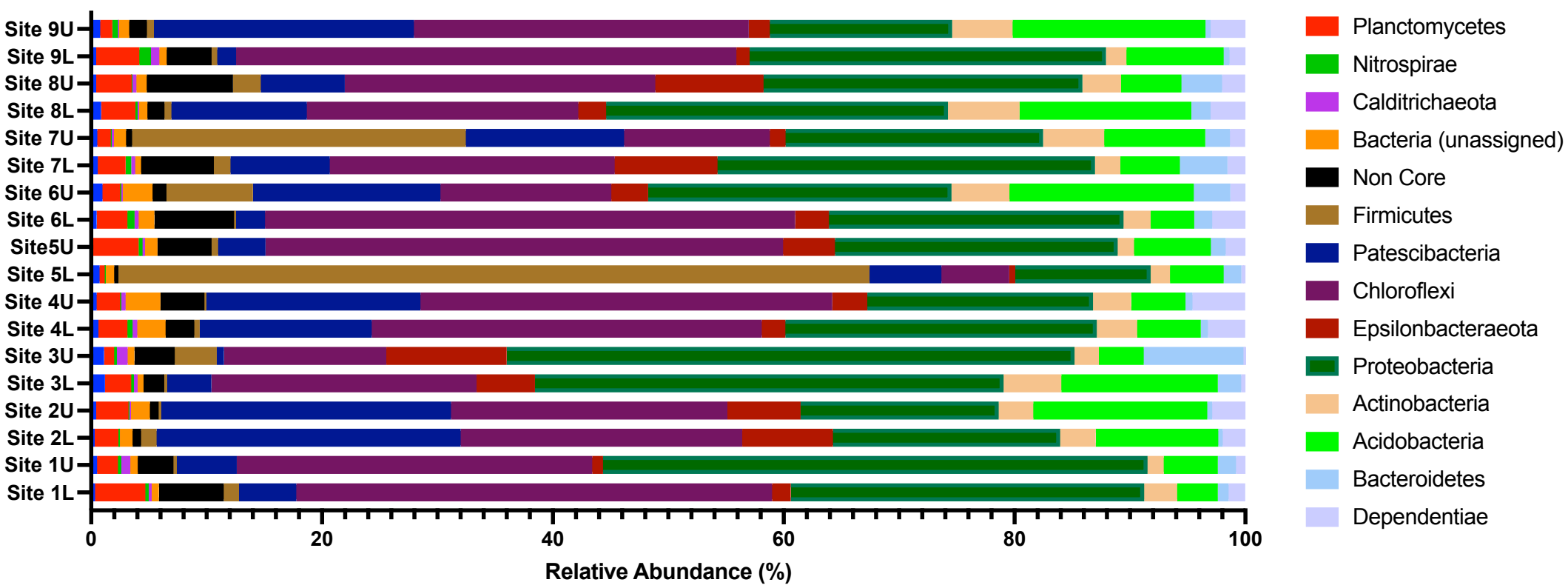

B

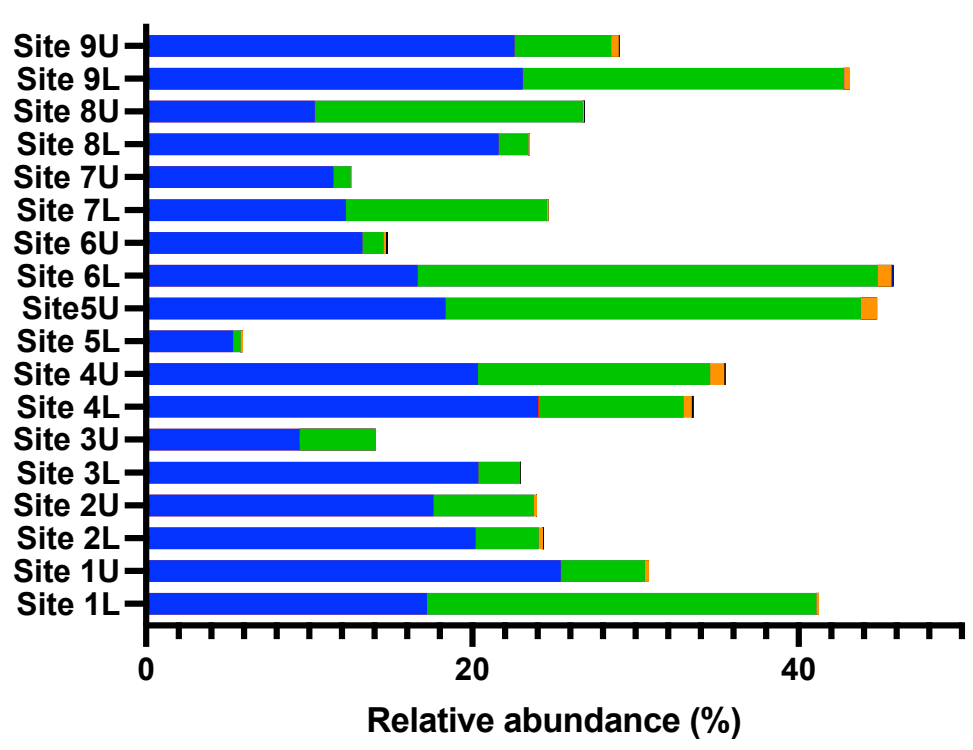

C

Anaerolineae

Chloroflexia

Dehalococcoidia

Gitt-GS-136

JG30-KF-CM66

KD4-96

OLB14

TK10

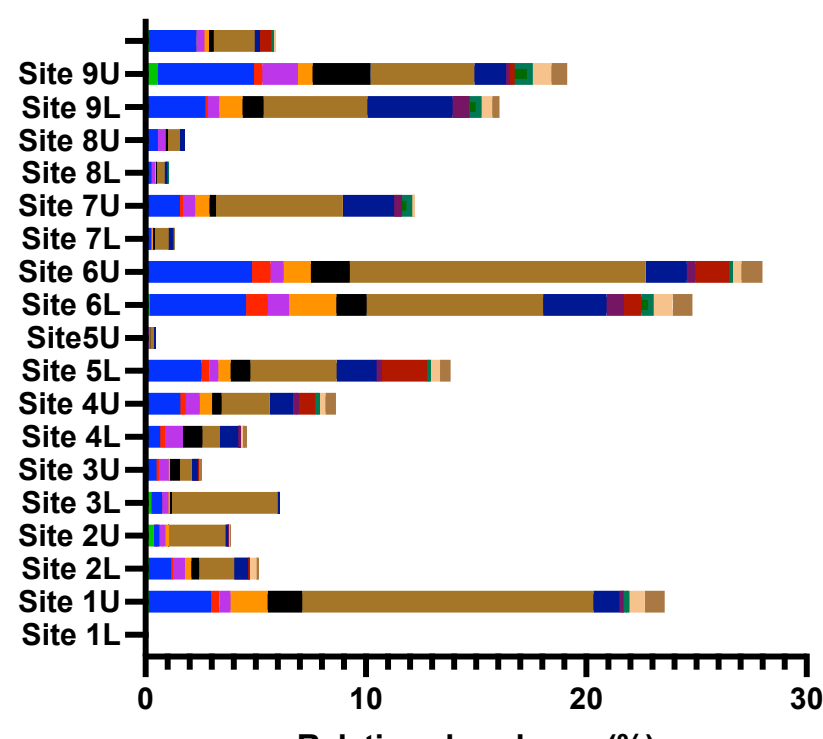

Dehalococcoidia

661239

Dehalococcoidales

FS117-23B-02

FW22

GIF3

GIF9

MSBL5

Napoli-4B-65

S085

Sh765B-AG-111

uncultured bacterium

vadinBA26 


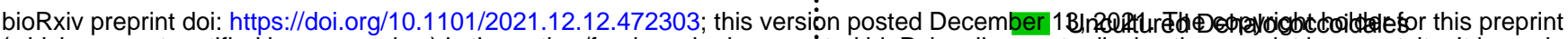
(which was not certified by peer review) is the author/funder, who has granted big Rxiv a license to display the preprint in perpetuity. It is made available under aCC-BY-NC 4.0 Inte national licenesehalobium
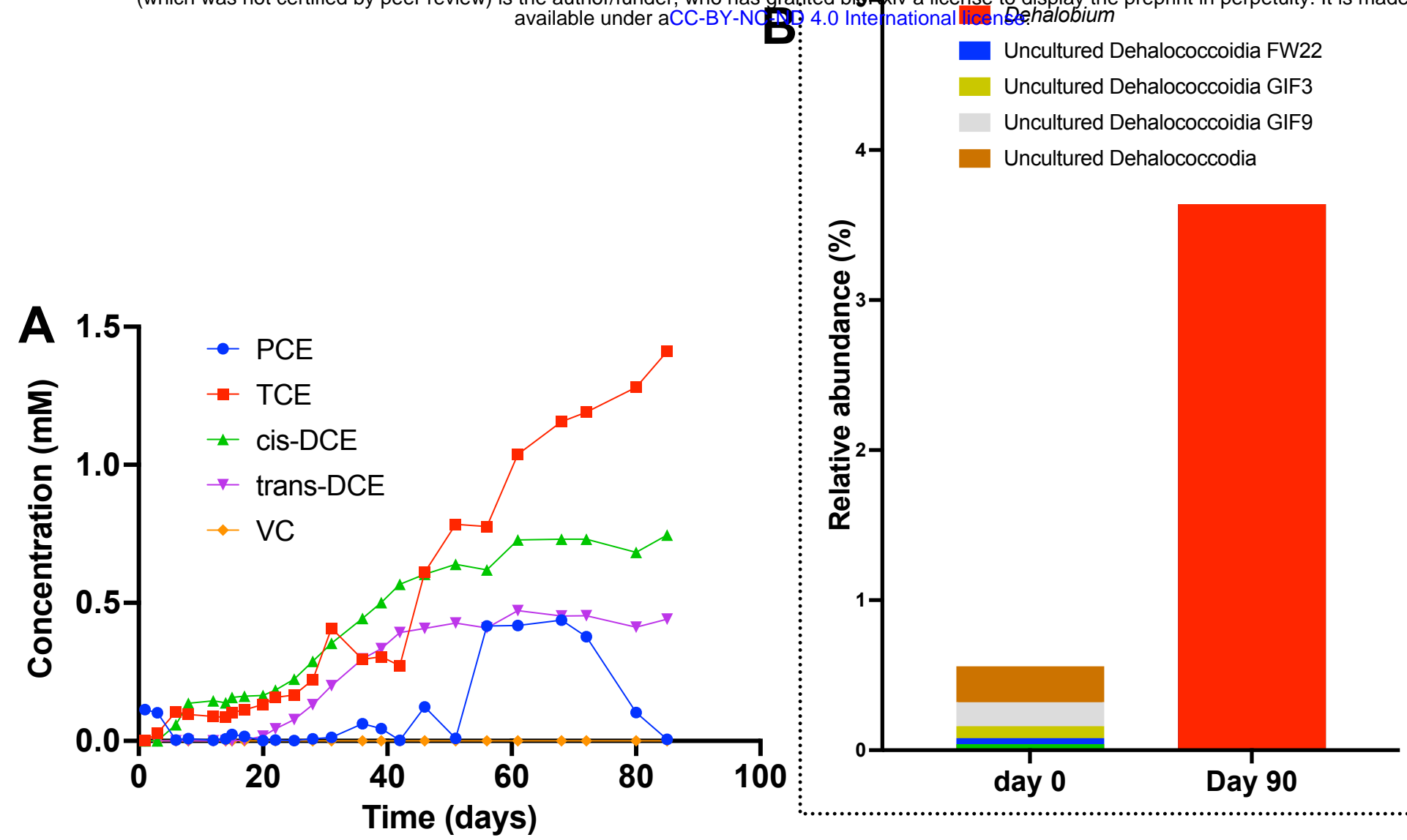

C

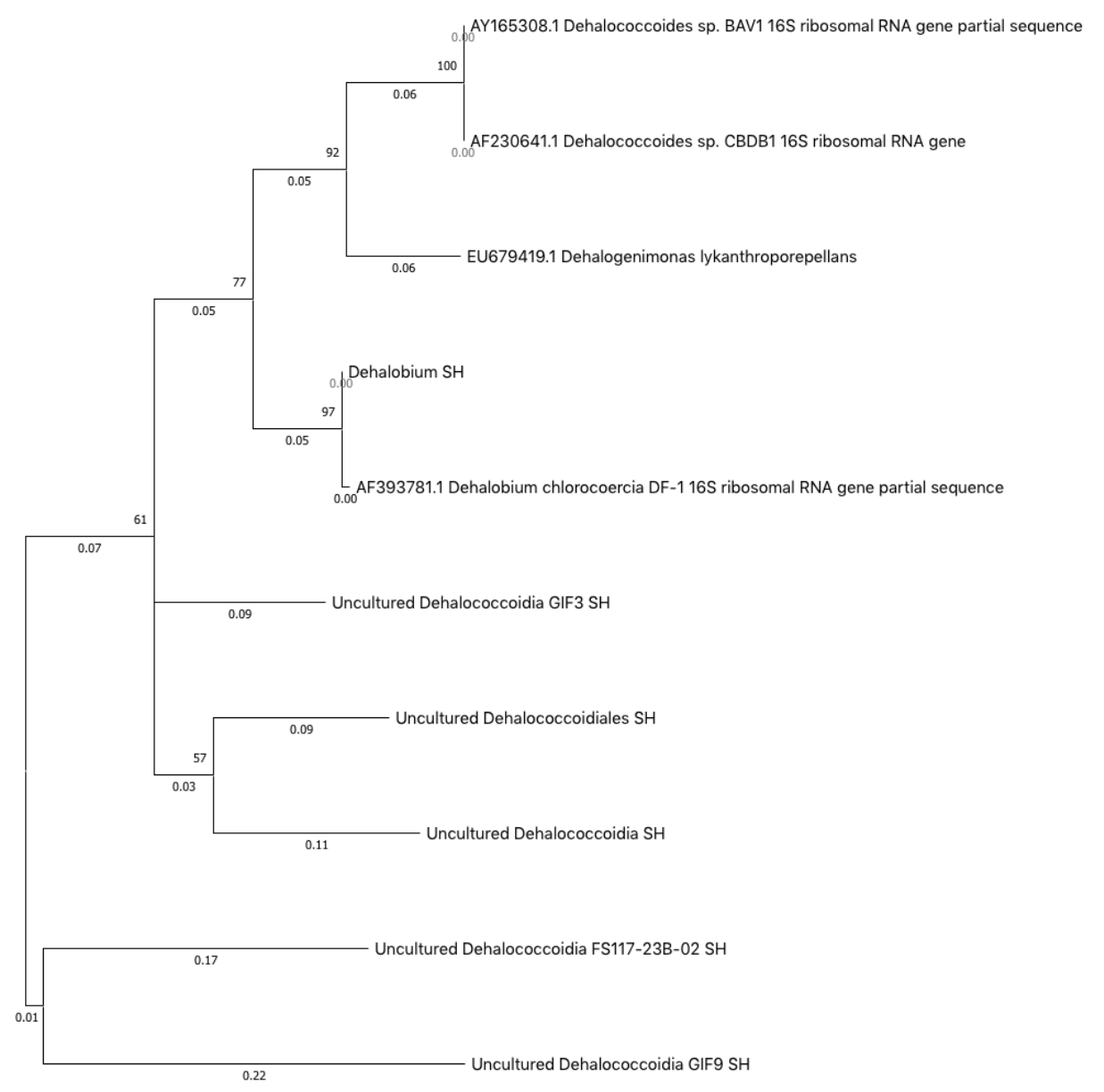



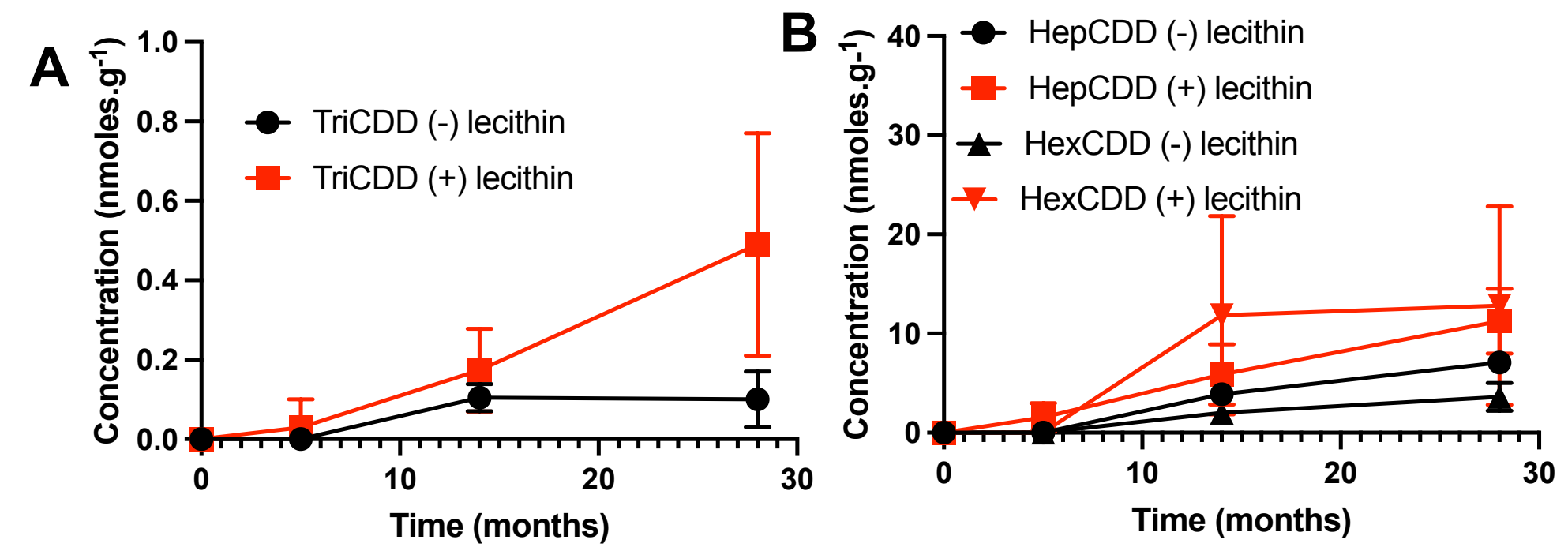

Class Level
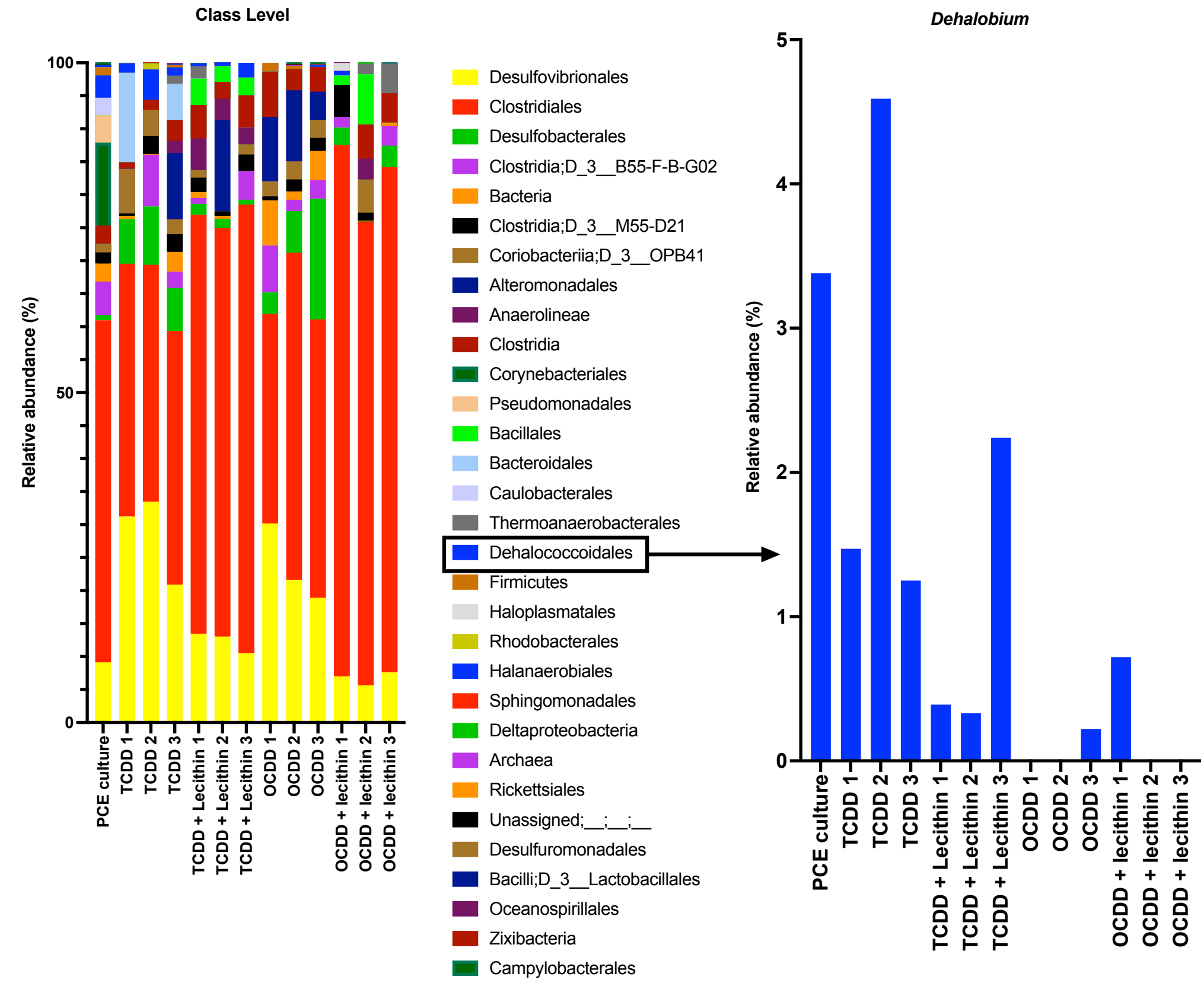

Dehalobium 